\title{
Aqueous phase transformation of glucose into HMF and levulinic acid combining homogeneous and heterogeneous catalysis
}

\author{
Diego Garcés, Laura Faba, Eva Díaz and Salvador Ordóñez*
}

\begin{abstract}
Homogeneous $(\mathrm{HCl})$ and heterogeneous catalysis (different kinds of zeolites) were combined for enhancing the glucose upgrading into two different platform molecules: 5-hydroxymethylfurfural (HMF) and levulinic acid (LA). $\beta$-zeolite was the most active material for the glucose isomerization to fructose, improving also the activity of $\mathrm{HCl}$ for the subsequent steps. Reaction time, temperature and $\mathrm{pH}$ were then modified, identifying $140^{\circ} \mathrm{C}$, $200 \mathrm{ppm}$ of $\mathrm{HCl}$ and $5 \mathrm{~h}$ as the optimum conditions for $\mathrm{HMF}$ formation ( $41 \%$ selectivity; $0.06 \mathrm{~mol} \cdot \mathrm{L}^{-1}$ ) and $140^{\circ} \mathrm{C}, 400 \mathrm{ppm}$ of $\mathrm{HCl}$ and $24 \mathrm{~h}$ for the levulinic acid ( $34 \%$ selectivity; $\left.0.12 \mathrm{~mol} \cdot \mathrm{L}^{-1}\right)$. This new approach is also relevant because of the significant changes on the mechanism of humins formation. All results are successfully fitted to a kinetic model simultaneously considering both catalytic mechanisms.
\end{abstract}

\section{Introduction}

In the frame of the circular economy, renewable carbon sources are of key interests. At this point, the transformation of sugars derived from cellulose and hemicellulose into valuable platform molecules, such as 5-hydroximethylfurfural (HMF) or levulinic acid (LA), is a required step to go forward in the development of new biodegradable polymers, drop-in biofuels and commodity chemicals. $^{[1]}$ HMF and LA are precursors of many different pharmaceuticals, personal care products, fragrances, resins, coatings, flavours, agro-chemical compounds, fuel additives, monomers and plasticizers. ${ }^{[2]}$ Both platform molecules are included in the top 10 list of the most valuable bio-based products from the carbohydrate biorefinery, according to the US Department of Energy. ${ }^{[3]}$ Their industrial value is also revealed in economic terms, with prices around $2500 € /$ ton and $5500 € /$ ton for HMF and LA, respectively, ${ }^{[4]}$ which also justifies the need of developing new strategies for their production in a cheaper and more sustainable way.

For manufacturing these platform molecules, a common first step, based on the hydrolysis of the stable and strong $\beta$-(1 $\rightarrow 4)$-glycosidic bonds of cellulose is required, obtaining the corresponding glucose molecules. ${ }^{[5]}$ Once the monomers are available, glucose can be transformed into LA or HMF by an acid-catalysed hydrolysis. Typically, these hydrolyses are carried out using homogeneous acids $\left(\mathrm{HCl}, \mathrm{H}_{2} \mathrm{SO}_{4}\right.$, etc.) in a multi-step reaction. ${ }^{[6,7]}$ Considering the reaction pathway

[a] D. Garcés, Dr. L. Faba, Dr. E. Díaz, Prof. S. Ordóñez. Department of Chemical and Environmental Engineering University of Oviedo, C/Julián Clavería $\mathrm{s} / \mathrm{n}-33006$ Oviedo, Asturias (Spain) Email: sordonez@uniovi.es

Supporting information for this article is given via a link at the end of the document. summarized in Scheme 1, levulinic acid (in equimolar quantities as formic acid) is produced by the rehydration and subsequent oxidation of HMF molecules, previously generated in the fructose dehydration. Fructose is obtained by isomerization of glucose molecules, being this step considered as a limiting step. According to these assumptions, reaction is limited by an inefficient isomerization (transformation of glucose into fructose) and the product selectivity is conditioned by the co-existence of aldehydes and acids in the medium, as well as undesired products obtained by lateral condensations of products and unreacted glucose (producing the generally known as humins) ${ }^{[8]}$ In particular, typical yields of 5-HMF reported in the literature are lower than $25 \%$, highlighting the low reaction rate and the large amount of anhydroglucose and glucose dimers, due to the presence of Brønsted acid catalysts. ${ }^{[9,10]}$ The direct dehydration of fructose to 5-HMF was also reported. [11] However, the relevance of this direct route is conditioned by the relative low-quantity available of this sugar. Glucose is the ideal raw material for the production of HMF since it is the most abundant and cheapest hexose. So, most of the current studies are focused on the development of new alternatives to make this reaction more attractive from the industrial point of view.

Higher selectivities to HMF have been reported using ionic liquid and organic solvents, such as DMA, MIBK, DMSO, DMF and $y$-valerolactone. ${ }^{[12,13]}$ Under these conditions, a common drawback related to the high affinity between HMF and those solvents was observed, requiring difficult purification steps that are not needed if the reaction is carried out in water. Solubility was taken into account in those studies proposing the use of two immiscible solvents, in such a way that the transference of HMF to the organic phase prevents its degradation into levulinic acid, reaction that only takes place in an aqueous medium. ${ }^{[14-16}$ However, the optimum approach should use water as solvent, that could be adapted for the productivity of one or other platform molecule as needed. Concerning the LA, more severe conditions are required (long reaction times, higher temperatures, lower $\mathrm{pH}$ ), being the selectivities typically conditioned by the large amount of humins produced at these conditions. ${ }^{[7,8]}$ With this premise, the main limiting factor is the good disposition of acid sites: Brønsted acid catalysts, such as $\mathrm{HCl}$, are required to promote dehydration and rehydration steps, whereas Lewis acids are needed to catalyse the isomerization from glucose to fructose. ${ }^{[17]}$ These sites are present in heterogeneous catalysts (zeolites, silico-alumiophosphate, AlMCM-41 and Al-MSU-F mesoporous materials and zirconia). The activity of these type of materials was previously reported in the literature obtaining promising productivities to HMF and levulinic acid. ${ }^{[18,19]}$ At these point, some authors have focused their efforts introducing different metals in the previously mentioned solid catalyst to improve their isomerization activity, obtaining relevant results combining tin in the large-pore beta (Sn-Beta). ${ }^{[18 b, 20]}$ The isomerization is clearly improved, but the activity for the other steps of the whole process decreases in comparison to the results previously reported with homogeneous catalysts.

In this work, a combination of homogeneous and heterogeneous catalysis for the glucose dehydration to HMF and levulinic acid is proposed, with the aim to optimize the kinetic of the process and to tune the reaction to the desired products, avoiding oligomers 


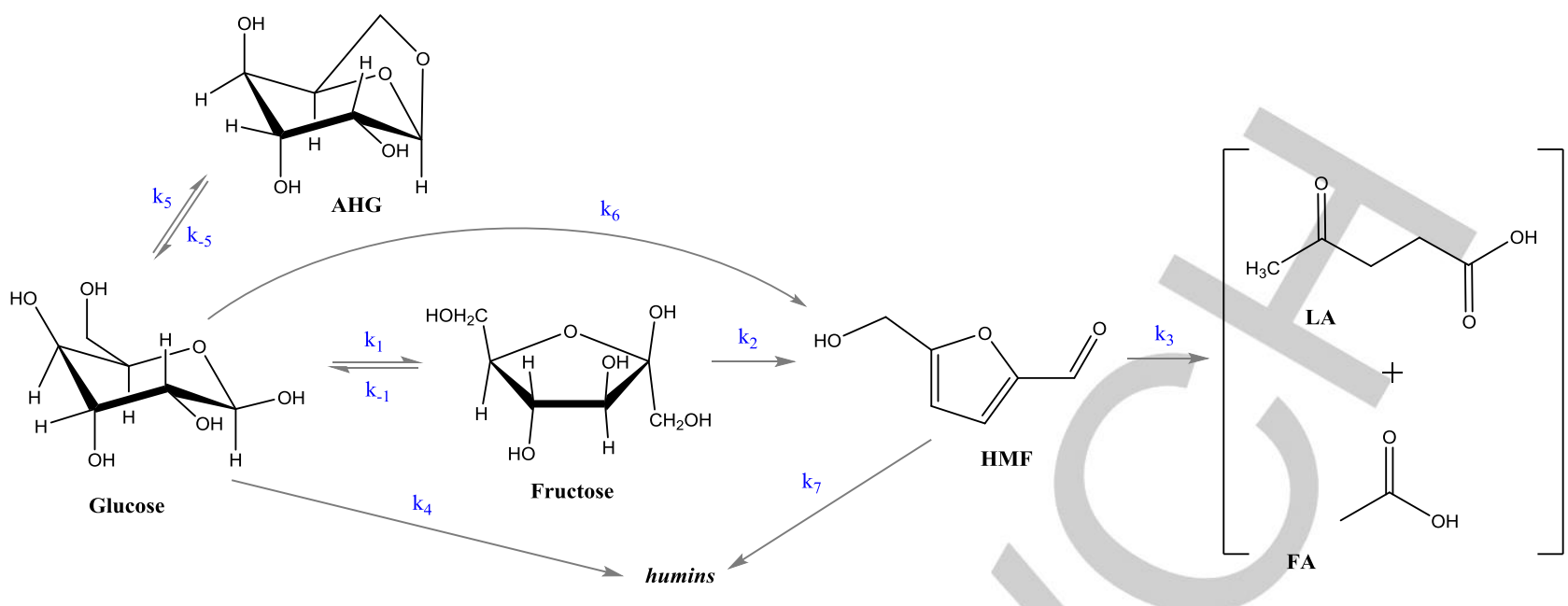

Scheme 1. Reaction pathway for the glucose valorization in aqueous media, as well as the kinetic constants identification

that can be produced by non-isomerized glucose. $\mathrm{HCl}$ was chosen as homogeneous catalyst, based on the well-known activity of this acid, its wide availability and the low cost of this chemical, being considered nowadays as a typical waste in many different industrial processes. ${ }^{[6]}$ Concerning the heterogeneous material, zeolites were considered because of their acid character (with important contribution of Lewis acidity), high hydrothermal stability and the easiness of regeneration. ${ }^{[15]}$

\section{Results and Discussion}

The effect of combining homogeneous and heterogeneous catalysis is analysed in Figure 1, comparing the temporal evolution of glucose conversion as function of the solid catalyst (ZSM-5, beta zeolite and $Y$ zeolite), working in presence of an acid solution $(\mathrm{HCl})$. Reaction conditions $(313 \mathrm{~K}, 0.185 \mathrm{M}$ of glucose, $200 \mathrm{ppm}$ of $\mathrm{HCl}$ ) were optimized in our previous work for the homogeneous reaction, ${ }^{[21]}$ whereas $2.66 \mathrm{~g} / \mathrm{L}$ of solid catalyst was added to each reaction. The presence of these heterogeneous catalysts does not affect to the global $\mathrm{pH}$, being constant at 2.03. This fact is justified by the strong acid character of $\mathrm{HCl}$ that prevails over the effect of any other weak acid introduced in the medium. Keeping constant the $\mathrm{pH}$, differences in terms of conversion and selectivities can be directly related to the influence of each solid catalyst. Concerning the glucose conversion, no effect of adding ZSM-5 was observed, obtaining a similar behaviour than using only the homogeneous catalyst: a fast conversion in the first two hours (around $20 \%$ ) and a softer increase in the following $22 \mathrm{~h}$ to reach a final conversion of 48.8 and $51.2 \%$, with and without ZSM-5, respectively. On the other hand, a clear improvement is observed in presence of beta zeolite, reaching almost complete conversion after $24 \mathrm{~h}(94.3 \%)$. This conversion is considerably higher than typical data reported for this reaction using heterogeneous materials, even at higher temperatures, ${ }^{[19}$ corroborating the synergetic effect of combining homogeneous and heterogeneous catalysis. Y-zeolite presents an intermediate behaviour, with a parallel evolution to that of the ZSM-5 during the first 12 hours, but without reaching the stationary state, extended the reaction to a final conversion of $67.2 \%$ after $24 \mathrm{~h}$.

In order to determine the role of the heterogeneous catalyst in the reaction products distribution, the selectivities of main compounds involved the reaction (fructose, anhydroglucose (AHG), 5-HMF and levulinic acid) as function of the glucose conversion are plotted in Figure 2. Formic acid was observed in equimolecular amount as levulinic acid, so its profiles are not included. Similar behaviour was obtained with only mineral acid, and combining $\mathrm{HCl}$ with ZSM-5 and $\beta$-zeolite. Fructose follows a typical profile of an initial reactive intermediate, with initial selectivities of $100 \%$ at differential conversion and a continuous decrease at increasing conversions. AHG and HMF show profiles congruent with primary products produced by parallel routes. The decreasing trend of HMF is congruent with its degradation into the corresponding acids (behaviour mainly observed with the beta zeolite) whereas AHG seems to reach a constant value, typical of a side product not undergoing further reactions. Finally, levulinic acid is a final product, with a continuous increase trend and a clear induction time.

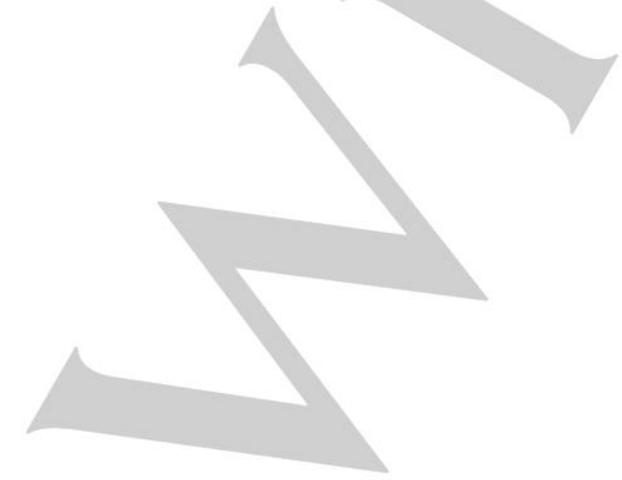



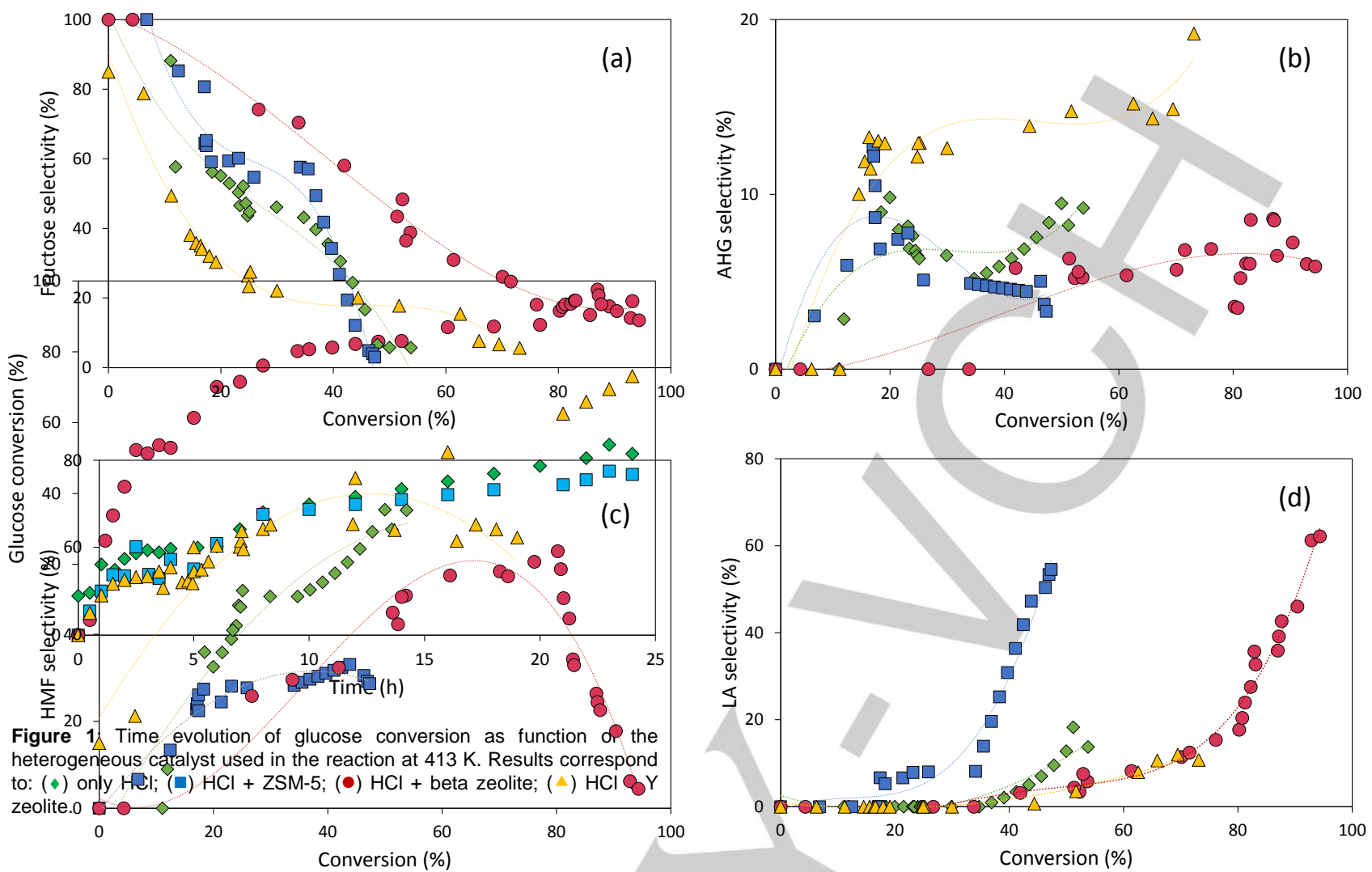

Figure 2. Selectivity evolution of main compounds involved glucose valorization at $413 \mathrm{~K}$ : (a) fructose; (b) AHG; (c) HMF; (d) Levulinic acid. Results as function of the catalyst: $(\bullet)$ only $\mathrm{HCl} ;(\square) \mathrm{HCl}+\mathrm{ZSM}-5 ;(\bullet) \mathrm{HCl}+$ beta zeolite; $(\Delta) \mathrm{HCl}+\mathrm{Y}$ zeolite.

Results obtained in absence of heterogeneous catalyst are conditioned by the low glucose conversion and the low levulinic acid production (final selectivity lower than $14 \%$ ), observing a continuous increase of HMF selectivity, reaching values higher than $68 \%$. Similar profiles were observed with ZSM-5 and beta zeolites, with higher selectivities to final acids, highlighting the results obtained with the second one (final selectivity higher than $62 \%$ for levulinic acid with almost total conversion of glucose). With ZSM-5, this selectivity is close to $54 \%$, but the lower conversion restricts its productivity. The high selectivities for HMF formation at high reactant conversions are also remarkable (70\% of selectivity at $80 \%$ of conversion, whereas in absence of heterogeneous catalysts, this value of the selectivity is obtained at a $40 \%$ of conversion).

A different evolution is observed with the $\mathrm{Y}$-zeolite. With this material, total selectivity to fructose formation is not observed at the lowest reaction time. This is congruent with the profiles observed for the HMF, with initial selectivity of $18 \%$. According to these results, a relevant direct dehydration of glucose is proposed when $\mathrm{Y}$-zeolite is used, without requiring the previous isomerization. This phenomenon has been previously reported in the literature when using other homogeneous catalysts, ${ }^{[8,10]}$ concluding that this dehydration is usually negligible in comparison to the dehydration of fructose. Despite this advantage, this material was discarded because of its low levulinic acid and the high AHG selectivity.

Humins were not quantified in the previous analysis because of the huge amount of different polymers that could be obtained from these compounds, both soluble and insoluble ones. Thus, this formation will be analysed in terms of decrease in the carbon balance, assuming that all the compounds of the main pathway were identified and all the lack of carbon is due to the formation of these oligomers. The carbon balance evolution is analysed in Figure 3. A parallel behaviour is observed for reaction catalysed by $\mathrm{HCl}$ and the combination of this mineral acid with Y-zeolite, with a continuous slight decrease, reaching final values close to $60 \%$. On the other hand, the carbon balance obtained with ZSM-5 (always higher than $74 \%$ ) suggests a lower humins productivity with this material, whereas a more relevant decrease is observed with the beta zeolite, obtaining a stable value at $42 \%$. The lack of correspondence between this profile and the levulinic acid one discards the degradation of acids and suggests that humins are produced by different oligomerizations of previous intermediates. If the carbon balance closure is analysed in detail for the reaction performed in presence of zeolite beta, it is observed that carbon balance is close to $100 \%$ at the initial reaction times, when fructose is the main reaction product, and HMF concentration is still low. When HMF selectivity increases, carbon mass balance closure starts to decrease, suggesting that HMF is the main humin precursor. On the contrary, glucose is considered as the main humins precursor in the case of homogeneous acid catalysed reactions, ${ }^{[8,10,22]}$

The low conversion obtained with only $\mathrm{HCl}$ and the $\mathrm{Y}$-zeolite is congruent then with the continuous decrease in their carbon balance profiles, suggesting humins production as a competitive reaction with the main pathway. However, this explanation is not congruent with the stable carbon balance profile obtained with ZSM-5, despite having final conversion lower than $50 \%$. The ZSM-5 profile must be explained according to the morphological 


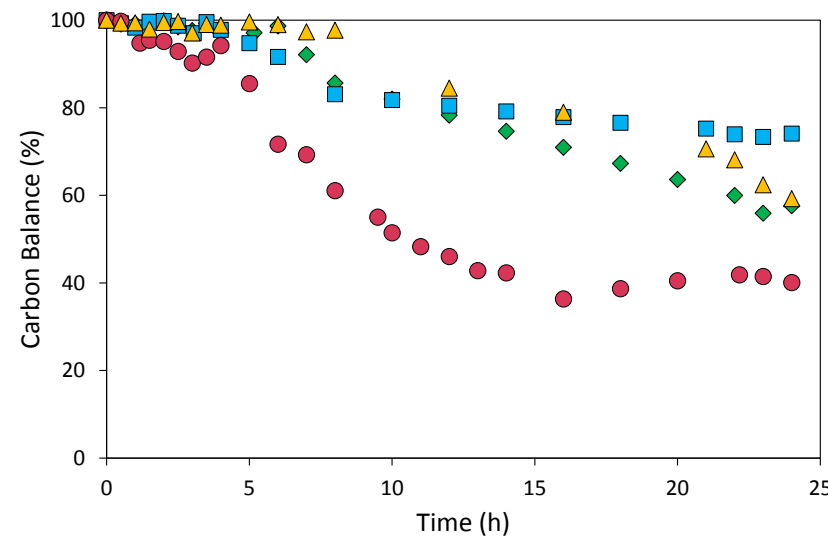

Figure 3: Evolution of carbon balance as function of the heterogeneous catalyst used in the reaction at $413 \mathrm{~K}$. Results correspond to: ( $\bullet$ ) only

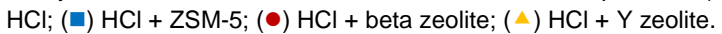

and chemical surface properties, summarized in Table 1. The low pore diameters of all these zeolites structures discard the oligomerization inside their structures, suggesting that these reactions are produced in the external surface or in the aqueous phase. The lower concentration of Lewis acid sites of ZSM-5 in comparison to the other solids is then proposed as the main cause of the low humins production observed with this material.

The expected improvement obtained by combining homogeneous and heterogeneous catalysis is better observed in terms of reaction kinetics. According to the literature, Lewis acidity from solid catalysts is required to promote the glucose isomerization to fructose. ${ }^{[17]}$ However, the subsequent reactions from fructose are catalysed by Brönsted acids. ${ }^{[17]}$ The presence of a mineral acid promotes them, displacing the first equilibrium, as well as the Brönsted acid of these zeolites. As consequence, all the steps are affected by the presence of heterogeneous materials and the reaction must be analysed globally without being possible to isolate the role of zeolites in this reaction.

This reaction involves both, liquid and solid phases. Thus, mass transfer processes must be considered for a complete kinetic analysis. A negligible effect of both, external and internal, mass transference limitation is assumed since the fast stirring (turbulent flow regime) and the small catalytic particle size (50$80 \mu \mathrm{m})$. These hypotheses were checked as function of two common criteria: the Wheeler-Weisz criteria and the Carberry number, for internal and external mass transferences, respectively. ${ }^{[25]}$ According to these methodologies, values higher than 0.1 and 0.05 are related to internal and external mass transference limitations, whereas their effect can be considered as negligible for lower values. As it was expected, experimental values are several orders of magnitude lower than these maximums: $1.2 \cdot 10^{-7}$ and $3 \cdot 10^{-5}$, respectively

Despite a Langmuir-Hinshelwood model based on adsorption equilibria could be considered (assuming a kinetic control based on the compounds adsorbed on the catalytic surface), power law kinetic models are more commonly used for aqueous-phase reactions. ${ }^{[10]}$ This approach also allow determine the homogeneous and heterogeneous contribution to the global kinetic. According to the most accepted pathway (Scheme 1), individual kinetic expressions must be considered since different kinetic orders are reported for each individual step. Thus, the glucose isomerization to fructose is a reversible reaction, with a first order dependence. ${ }^{[10]}$ Dehydration of fructose to HMF is considered as an irreversible reaction, kinetically conditioned by the enol formation, and with subsequent first order dependence. ${ }^{[10,26]}$ Different researchers conclude that direct dehydration of glucose into HMF is also possible, but the typical kinetics is significantly slower than the corresponding to dehydration via previous isomerization. ${ }^{[27,28]}$ The levu-linic and formic acids are stoichiometrically obtained by the HMF through a first-kinetic order. ${ }^{[29}$

Finally, two side reactions must be considered. On the one hand, glucose undergoes intramolecular dehydration in acid medium, yielding anhy-droglucose. This reaction is considered in the literature as a first-order reversible one. ${ }^{[30]}$ On the other hand, there is not a good agreement about the humins production, being their formation reported from glucose and polymerization reactions, but also from HMF or by reaction intermediates, such as the formic acid. ${ }^{[6,11,31,32]}$ As the ratio formic acid to levulinic acid keeps almost constant and equimolecular during all the experiments, and according to the carbon balance obtained with the beta zeolite, this last option has been discarded. So, the formation from both glucose and HMF is considered as routes for humins formation. In this case, a first order dependence can be discarded because of the polymerization character, involving more than one molecule. According to the previous results reported in the literature, and because of the best fitting with experimental results, a second kinetic order was considered. ${ }^{[4,10]}$ All these theoretical premises were experimentally confirmed analysing the profiles obtained when using different intermediates as reactants. Temporal profiles are included in Figure 4. It is observed that when fructose is used as a reactant, significant amounts of glucose were produced, suggesting the reversibility of the glucose-fructose reaction, whereas glucose is also observed when using anhydroglucose as reactant. Concerning to the two main reaction products, LA was observed to be a stable final product, whereas HMF reaction leads to LA and humins.

According to these considerations, proposed kinetic model is summarized in the following equations, where " $G$ " indicates glucose; "F", fructose; "A" anhydroglucose; "H", humins; "LA", levulinic acid; and "FA", formic acid.

$$
\begin{gathered}
\frac{d G}{d t}=-k_{1} \cdot G-k_{4} \cdot G^{2}-k_{5} \cdot G-k_{6} \cdot G+k_{-1} \cdot F+k_{-5} \cdot A \\
\frac{d A}{d t}=k_{5} \cdot G-k_{-5} \cdot A
\end{gathered}
$$

Table 1. Morphological and chemical properties of heterogeneous catalysts used in this work ${ }^{[23,24]}$

\begin{tabular}{|c|c|c|c|c|c|c|c|c|c|}
\hline \multirow{2}{*}{ Catalyst } & \multirow{2}{*}{$\mathrm{S}_{\mathrm{BET}}\left(\mathrm{m}^{2} \cdot \mathrm{g}^{-1}\right)$} & \multirow{2}{*}{$\mathrm{Vp}\left(\mathrm{cm}^{3} \cdot \mathrm{g}^{-1}\right)$} & \multirow{2}{*}{$\mathrm{d}_{\mathrm{p}}(\AA)$} & \multicolumn{3}{|c|}{ Lewis acidity $\left(\mu \mathrm{mol} \cdot \mathrm{g}^{-1}\right)$} & \multicolumn{3}{|c|}{ Brönsted acidity $\left(\mu \mathrm{mol} \cdot \mathrm{g}^{-1}\right)$} \\
\cline { 5 - 10 } & & & & Weak & Medium & Strong & Weak & Medium & \multicolumn{2}{|c|}{ Strong } \\
\hline ZSM-5 & 443 & 0.301 & 5.1 & 36 & 8 & 3 & 416 & 395 & 285 \\
\hline Beta & 589 & 0.409 & 6.7 & 82 & 43 & 25 & 219 & 187 & 125 \\
\hline $\mathrm{Y}$ & 884 & 0.47 & 7.4 & 123 & 75 & 58 & 255 & 205 & 129 \\
\hline
\end{tabular}




$$
\begin{gathered}
\frac{d F}{d t}=k_{1} \cdot G-k_{-1} \cdot F-k_{2} \cdot F \\
\frac{d H}{d t}=k_{2} \cdot F+k_{6} \cdot G-k_{3} \cdot H-k_{7} \cdot H^{2}
\end{gathered}
$$
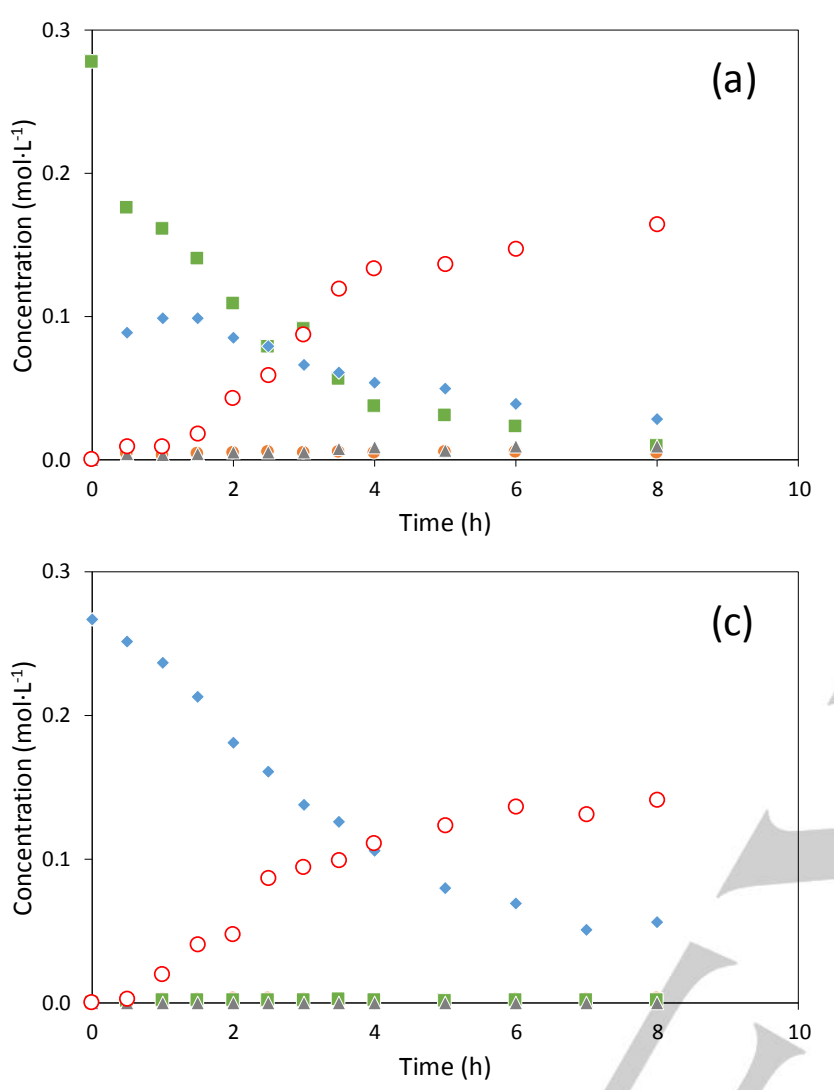

factors higher than 0.96 in all the cases. It must be highlighted that, in good agreement with the initial hypothesis, all these coefficients are higher than the previously reported ones considering a kinetic model based on adsorption equilibria. ${ }^{[3]}$ Concerning the analysis step-by-step, the positive effect of combining heterogeneous and homogeneous catalysts is in
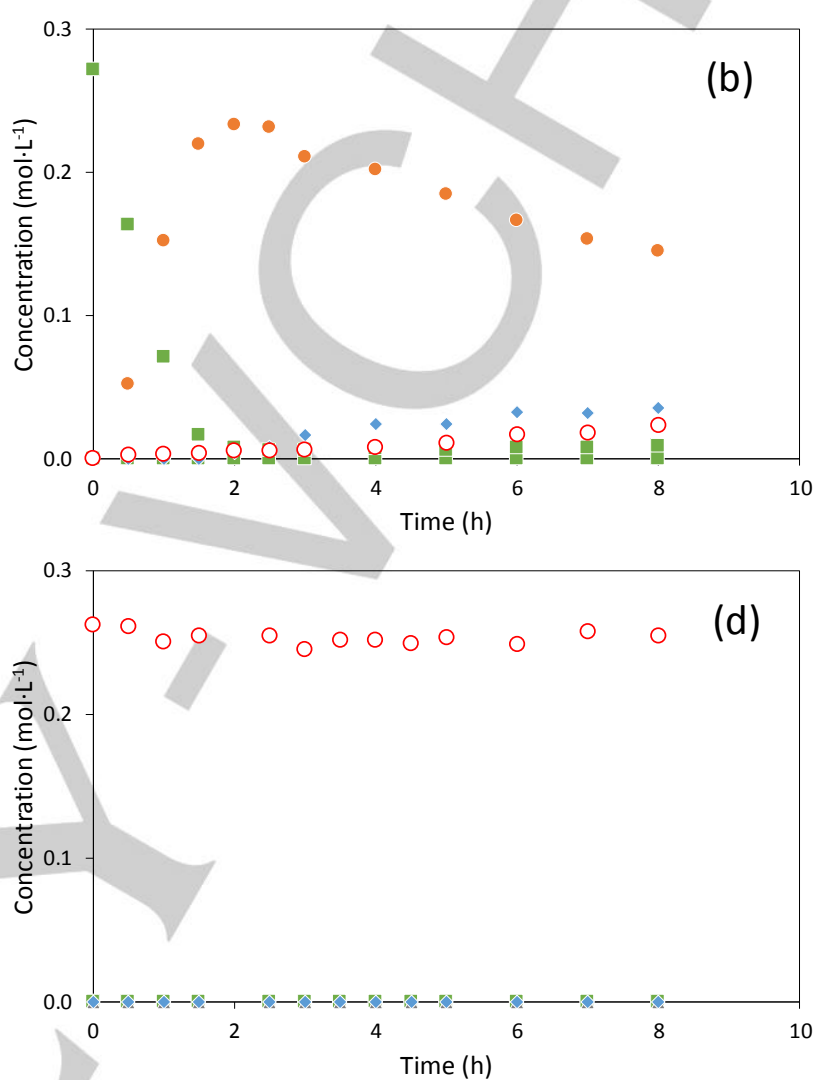

Figure 4. Temporal profiles obtained when (a) fructose; (b) AHG; (c) HMF and (d) LA as reactants in the reaction catalyzed by beta zeolite and 200 ppm of $\mathrm{HCl}$ at $413 \mathrm{~K}$. Symbols: $(\bullet)$ glucose; ( $(-)$ fructose; $(\bullet) \mathrm{HMF} ;(\triangle)$ AHG; (o) LA.

$$
\frac{d L A}{d t}=\frac{d F A}{d t}=k_{3} \cdot H
$$

It must be highlighted that all these constants are apparent constants, in which the role of $\mathrm{HCl}$ in the kinetic model is also included. Taking into account that all these experiments were carried out with the same $\mathrm{HCl}$ concentration, this assumption can be done. The corres-pondence among experimental and model prediction for the reactions performed in absence of heterogeneous catalyst and in presence of the three zeolites proposed in this work is observed in the lines of Figure 5, whereas the kinetic constants are summarized in Table 2.

The goodness of this model is clearly observed, with correlation agreement with the increase in all the direct reactions, with main effect observed when using the beta zeolite. With this material, the fructose reverse isomerization is also reduced, with the corresponding effect in the global results. The levulinic acid production step is also promoted with this zeolite, but experimental results are mainly conditioned by the second step of the process: the fructose dehydration to obtain HMF. This step is strongly improved when using the $\beta$-zeolite, but is almost prevented with the other two zeolites. Considering that same amount of $\mathrm{HCl}$ is presented in all the experiments, an improvement was expected when a second acid material is added to the system. However, same type of acidity is responsible of undesired reactions, such as the humins production and the glucose stabilization. In addition, zeolites

\begin{tabular}{|c|c|c|c|c|c|c|c|c|c|c|c|c|}
\hline \multirow{2}{*}{$\begin{array}{c}\mathrm{HCl} \\
(\mathrm{ppm})\end{array}$} & \multirow{2}{*}{$\begin{array}{l}\text { Heter. } \\
\text { Catalyst }\end{array}$} & \multirow{2}{*}{$\begin{array}{c}\mathrm{T} \\
(\mathrm{K})\end{array}$} & \multicolumn{9}{|c|}{ Kinetic constants (units in $\mathrm{h}^{-1}$ except $\mathrm{k}_{4}$ and $\mathrm{k}_{7}$ that are in $\mathrm{L} \cdot \mathrm{mol}^{-1} \cdot \mathrm{h}^{-1}$ ) } & \multirow[t]{2}{*}{$r^{2}$} \\
\hline & & & $\mathrm{k}_{1}$ & $\mathrm{k}_{-1}$ & $\mathrm{k}_{2}$ & $\mathrm{k}_{3}$ & $\mathrm{k}_{4}$ & $\mathrm{k}_{5}$ & $\mathrm{k}_{-5}$ & $\mathrm{k}_{6}$ & $\mathrm{k}_{7}$ & \\
\hline 200 & & 413 & $0.19 \pm 0.03$ & $1.55 \pm 0.47$ & $0.19 \pm 0.03$ & $0.01 \pm 0.01$ & $0.40 \pm 0.07$ & $0.002 \pm 0.001$ & $0.10 \pm 0.04$ & $0.02 \pm 0.01$ & $10.81 \pm 1.41$ & 0.994 \\
\hline 200 & ZSM-5 & 413 & $0.28 \pm 0.14$ & $3.01 \pm 1.26$ & $0.04 \pm 6 \cdot 10^{-9}$ & $0.02 \pm 0.02$ & $0.56 \pm 3 \cdot 10^{-7}$ & $0.01 \pm 8 \cdot 10^{-16}$ & $0.10 \pm 0.02$ & $0.03 \pm 2 \cdot 10^{-7}$ & $21.31 \pm 1.12$ & 0.992 \\
\hline 200 & Y zeolite & 413 & $0.17 \pm 0.10$ & $2.65 \pm 0.83$ & $0.02 \pm 0.01$ & $0.02 \pm 0.01$ & $0.53 \pm 0.13$ & $0.002 \pm 0.001$ & $0.07 \pm 0.05$ & $0.09 \pm 0.03$ & $19.97 \pm 2.19$ & 0.994 \\
\hline 200 & $\beta$ zeolite & 413 & $0.43 \pm 0.01$ & $0.14 \pm 0.10$ & $0.69 \pm 0.10$ & $0.08 \pm 0.02$ & $1.13 \pm 0.08$ & $0.01 \pm 0.01$ & $0.19 \pm 0.14$ & 0 & $10.27 \pm 0.41$ & 0.97 \\
\hline
\end{tabular}

Table 2. Kinetic constants obtained after fitting the experimental results to the model proposed. Results corresponding to the different catalytic systems studied in this work 
have different behaviours as function of their internal structure and this acidity cannot be entirely active for molecules as big as these sugar derivatives. Moreover, it is known than Y-zeolite is not entirely stable in aqueous phase, and surface modifications can affect to the exposed acidity. As conclusion of the first experiments, $\beta$-zeolite is chosen as the best heterogeneous catalyst, despite the lower carbon balance obtained with this material (kinetically explained by the promotion of humins
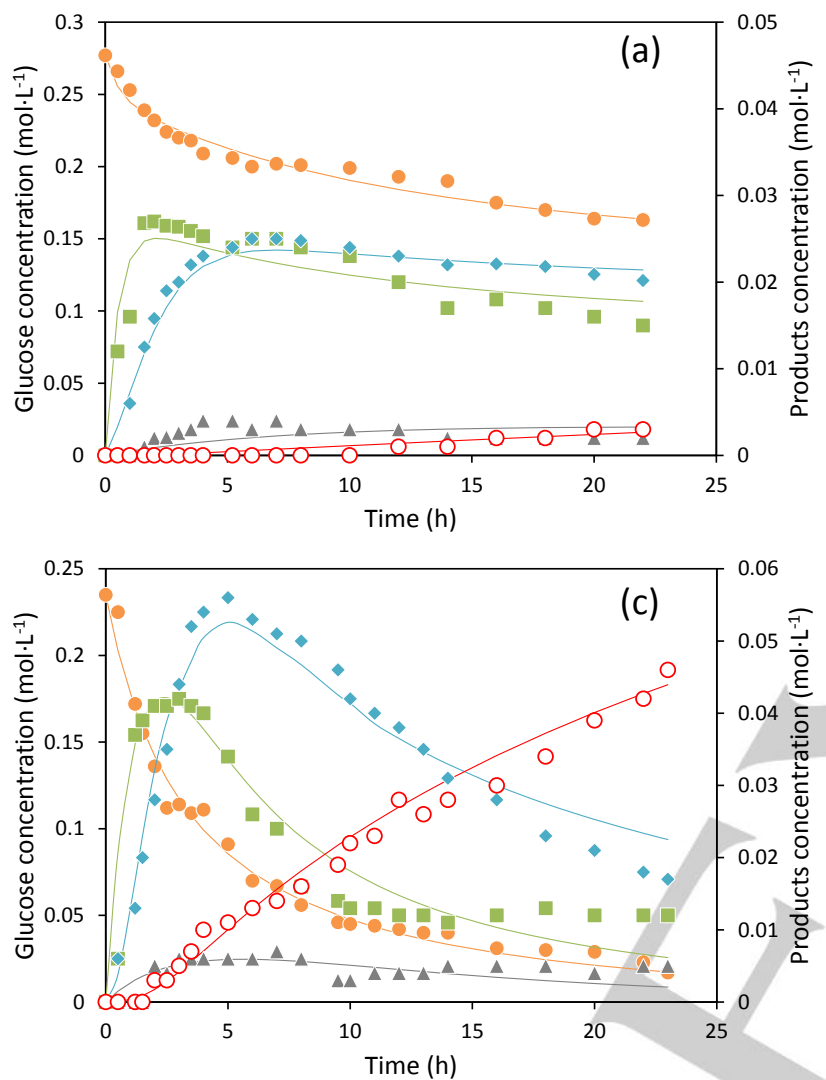

obtained at softer temperatures (lower than $45 \%$ in both cases). Similar behaviour is obtained for the levulinic acid. In this case, this component is promoted at longer reaction times, in such a way that the selectivity after $24 \mathrm{~h}$ increases, for values around $37-40 \%$ at 393 and $403 \mathrm{~K}$ to more than $62 \%$ at $413 \mathrm{~K}$.

As function of these results, the initial temperature $(413 \mathrm{~K})$ is chosen as the optimum one for both components, setting the time to the desired product. These results can be explained with evolution of the kinetic constants (the correspondence among
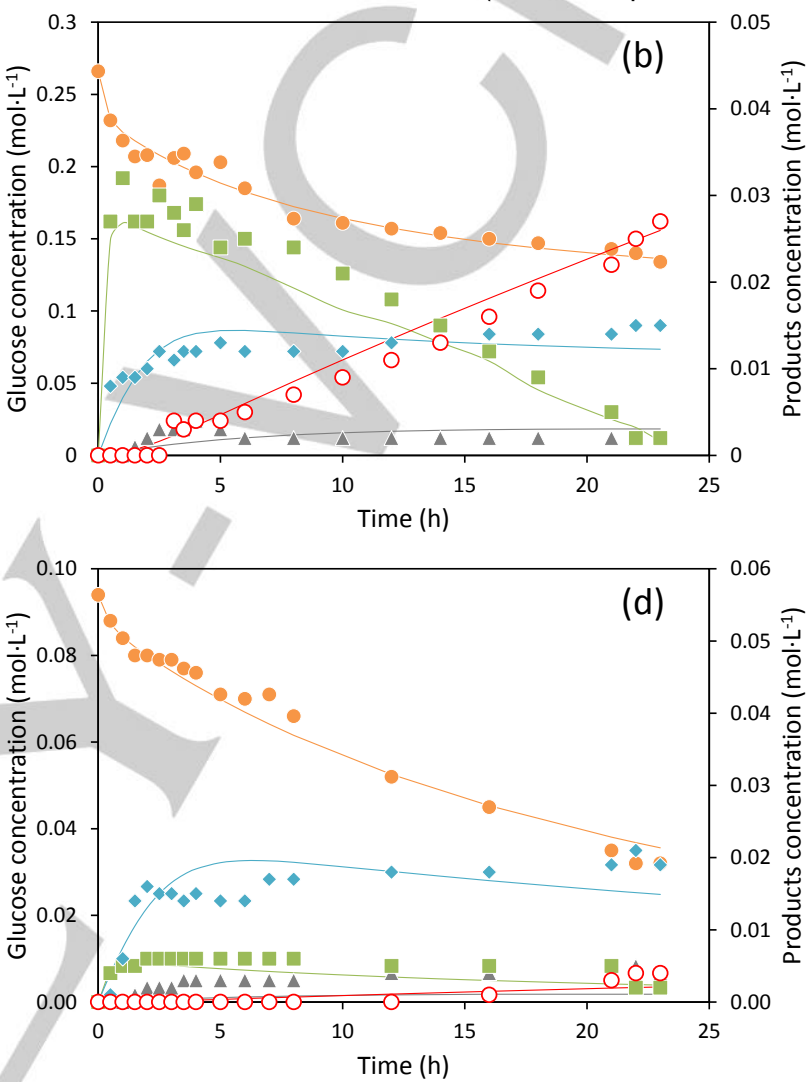

Figure 5: Experimental (points) and fitted values (lines) obtained according to the kinetic model proposed for the glucose conversion at $413 \mathrm{~K}$

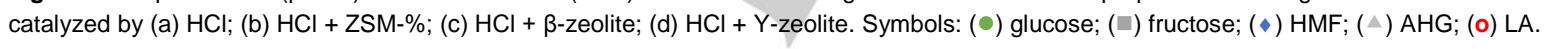

production, mainly from HMF).

Trying to reduce this degradation, the effect of temperature was analysed, carrying out same experiments at softer conditions. Main results can be observed in Figure 6, whereas the detailed profiles are included in the Supporting Information (Fig. S1 - S3) Concerning the glucose conversion, a clear decrease is observed at lower temperatures, obtained an almost parallel evolution for reactions at 393 and $403 \mathrm{~K}$. In all the cases, apparent irreversible first-order kinetics was observed. After $24 \mathrm{~h}$ reaction time, more than 54 and $71 \%$ of glucose disappearance was obtained at 393 and $403 \mathrm{~K}$, respectively. This decrease in the glucose conversion, however, does not correspond to an improvement in the final carbon balance, obtaining same decreasing trend and similar final values between 47 and $51 \%$. According to these results, the humins formation cannot be prevented by decreasing the temperature.

Concerning the most relevant products, both are preferentially obtained at the highest temperature tested. The maximum HMF productivity is reached after $5 \mathrm{~h}$, with a selectivity higher than $54 \%$. This value is significantly higher than the maximum ones experimental and fitted data is plotted in Fig. S1-S3 of supporting information).

According to results shown in Table 3, the expected improvement in the kinetic constants at increasing temperature is clearly observed, being this enhancement more evident in the case of the first step: the glucose isomerization. The reaction rate for this step sharply increases with the temperature, boosting the production of both desired platform molecules. An Arrhenius dependence was observed for the main reaction steps, obtaining the activation energies also included in Table 3 . The good fit of these constants and the Arrhenius model is included in the Supporting Information (Fig. S4). The important improvement obtained by combining homogeneous and heterogeneous catalysis is clearly observed by comparing these values with the previous ones reported in the literature. Almost all the activation energies are around 40 to $50 \%$ lower than values included in studies using only homogeneous catalysis, where the typical values are in the range $120-$ $160 \mathrm{~kJ} / \mathrm{mol}^{[1 \mathrm{1c}, 8,10,34,35]}$ In fact, these values are $30 \%$ than values corresponding to using acetone as solvent, one of the best alternatives for maximizing the 5-HMF under mineral acid 
conditions. ${ }^{[36]}$ The only exception is the $\mathrm{k}_{6}$ activation energy. This kinetic constant corresponds to the AHG production and the value obtained is quite similar to the proposed one using only $\mathrm{HCl}\left(138 \mathrm{~kJ} / \mathrm{mol}\right.$ in the literature ${ }^{[8]} 132.6 \mathrm{~kJ} / \mathrm{mol}$ in our results). Considering the negative role of this compound in the proposed aim, the lack of influence in this reaction is also an advantage that must be highlighted.

In order to determine the effect of the mineral acid on the reaction performance, experiments at different $\mathrm{HCl}$ concentration were carried out: 50, 200 and $400 \mathrm{ppm}$. An additional reaction was performed in absence of mineral acid (only beta zeolite, $\mathrm{pH}=4.92$ ) in order to compare the results obtained. In general terms, each kinetic constant (previously labelled as " $k_{i}$ " corresponds to the intrinsic " $k_{i}^{*}$ ", and a power-law
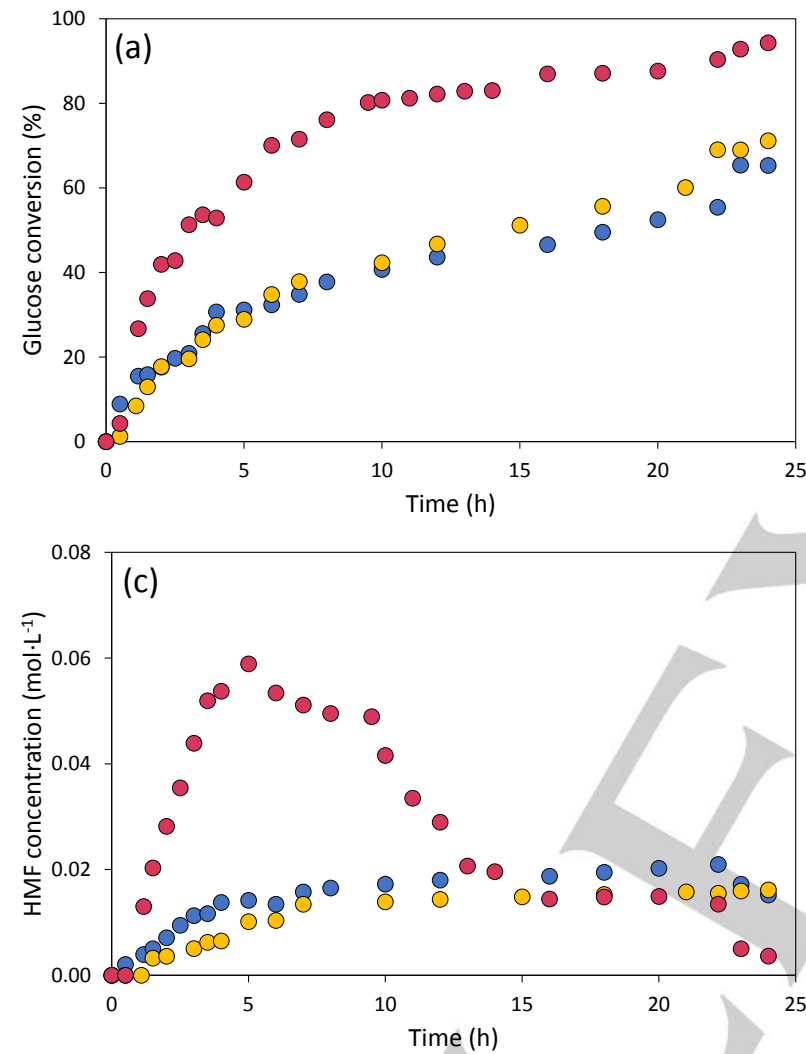

expression:

$$
k_{i}=k_{i}^{*} \cdot[H C l]_{i}^{m_{i}}
$$

This dependence has been previously proposed for kinetic studies controlled by homogeneous catalysis. ${ }^{[1,37]}$ Experimental data obtained with $0,50,200$ and $400 \mathrm{ppm}$ were fitted to the proposed model, being these profiles included in the Supporting Information (Fig. S5-S7, except in the case of $200 \mathrm{ppm}$, previously plotted in Fig. 6c). Table 4 summarizes the main results obtained, whereas the goodness of the proposed dependence of the kinetic constants on the $\mathrm{pH}$ is depicted in the supporting information (Fig. S8). The lowest value of the intrinsic constant is obtained for the first step, in good agreement with
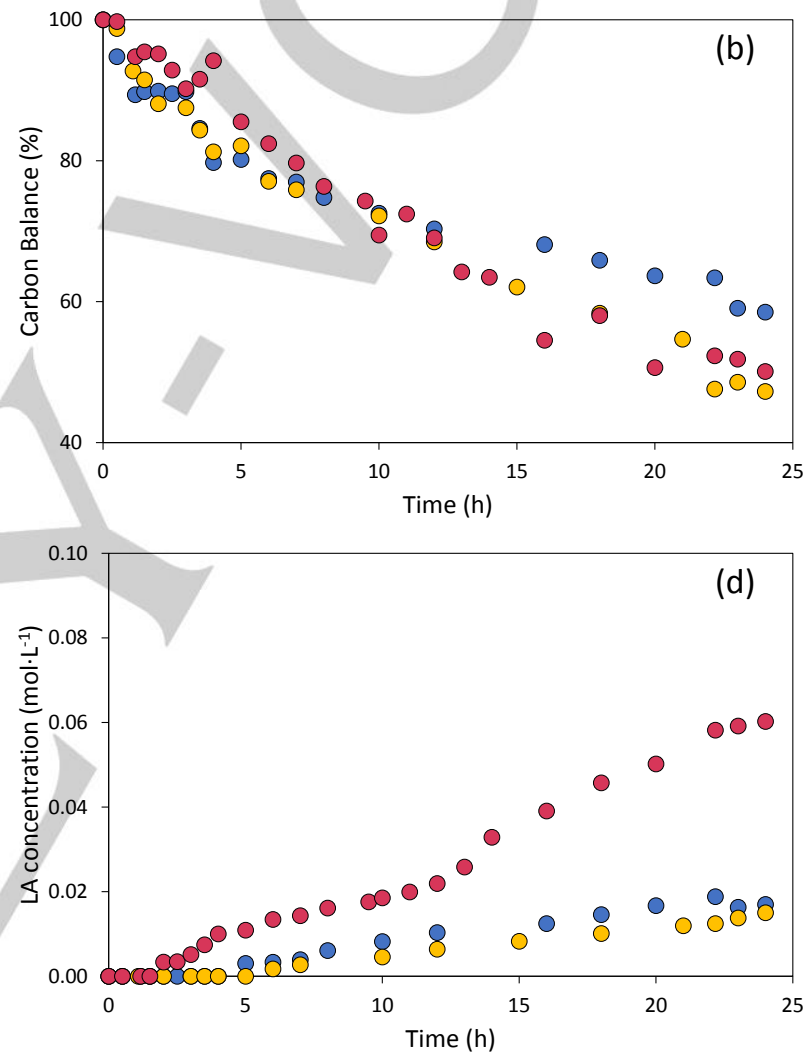

Figure 6: Effect of reaction temperature in the glucose valorization catalyzed by $\mathrm{HCl}+\beta$-zeolite when reaction is carried out at $393 \mathrm{~K}$ (blue); $403 \mathrm{~K}$ (yellow); and $413 \mathrm{~K}$ (red). Results analyzed in terms of: (a) glucose conversion; (b) carbon balance; (c) HMF concentration; (d) levulinic acid.

function of the $\mathrm{HCl}$ concentration, according to the following the previous hypothesis that this step is mainly catalysed by

Table 3. Kinetic constants obtained after fitting the experimental results at different temperatures to the model proposed and constants obtained applying an Arrhenius model

\begin{tabular}{|c|c|c|c|c|c|c|c|c|c|c|c|}
\hline \multirow{2}{*}{$\begin{array}{c}\mathrm{HCl} \\
(\mathrm{ppm})\end{array}$} & \multirow{2}{*}{$\begin{array}{l}\text { Heter. } \\
\text { Catalyst }\end{array}$} & \multirow{2}{*}{$\begin{array}{c}\mathrm{T} \\
(\mathrm{K})\end{array}$} & \multicolumn{8}{|c|}{ Kinetic constants (units in $\mathrm{h}^{-1}$ except $\mathrm{k}_{4}$ and $\mathrm{k}_{7}$ that are in $\mathrm{L} \cdot \mathrm{mol}^{-1} \cdot \mathrm{h}^{-1}$ ) } & \multirow[t]{2}{*}{$r^{2}$} \\
\hline & & & $\mathrm{k}_{1}$ & $\mathrm{k}_{-1}$ & $\mathrm{k}_{2}$ & $\mathrm{k}_{3}$ & $\mathrm{k}_{4}$ & $k_{5}$ & $\mathrm{~K}_{-5}$ & $\mathrm{k}_{7}$ & \\
\hline 200 & $\beta$ zeolite & 413 & $0.43 \pm 0.01$ & $0.14 \pm 0.10$ & $0.69 \pm 0.10$ & $0.08 \pm 0.02$ & $1.13 \pm 0.08$ & $0.01 \pm 0.01$ & $0.19 \pm 0.14$ & $10.27 \pm 0.41$ & 0.97 \\
\hline 200 & $\beta$ zeolite & 403 & $0.29 \pm 0.07$ & $0.56 \pm 0.12$ & $0.43 \pm 0.13$ & $0.08 \pm 0.03$ & $0.74 \pm 0.01$ & $0.02 \pm 0.001$ & $0.12 \pm 0.05$ & $5.41 \pm 0.52$ & 0.98 \\
\hline 200 & $\beta$ zeolite & 393 & $0.17 \pm 0.02$ & $2.74 \pm 0.08$ & $0.28 \pm 0.21$ & $0.05 \pm 0.01$ & $0.38 \pm 0.01$ & $0.002 \pm 0.001$ & $0.05 \pm 0.02$ & $3.97 \pm 0.34$ & 0.96 \\
\hline \multicolumn{3}{|c|}{ Pre-exponential factor } & $3.6 \cdot 10^{7}$ & - & $2.7 \cdot 10^{7}$ & $1.3 \cdot 10^{3}$ & $1.8 \cdot 10^{9}$ & $1.2 \cdot 10^{15}$ & - & $1.2 \cdot 10^{9}$ & - \\
\hline \multicolumn{3}{|c|}{ Activation Energy (kJ/mol) } & 62 & - & 60 & 33 & 72 & 132 & - & 64 & - \\
\hline \multicolumn{3}{|c|}{$r^{2}$} & 0.992 & - & 0.997 & 0.999 & 0.98 & 0.68 & - & 0.95 & - \\
\hline
\end{tabular}


Lewis acid sites. According to results obtained, the kinetic order of $\mathrm{HCl}$ is close to one in most of the individual steps (considering as first order those exponents from 0.5 to 1.1 , being these discrepancies justified by experimental errors), except in the case of the HMF oxidation to levulinic and formic acids, in which the power coefficient is higher. This unexpected value could indicate a stronger proton dependence for the HMF degradation, probably justified by the breakdown required (one HMF molecule will be divided into two acids) as well as the strong oxidation required). Hypothesis proposed is that this step is not really an elementary step but a set of experimental ones. This global sense is congruent with a higher kinetic order. In fact, values higher than 1 were previously obtained in kinetic studies about

$$
\begin{gathered}
C S F=\log \left(R_{0}\right)-p H \\
R_{0}=t \cdot \exp \left(\frac{T-T_{r e f}}{14.75}\right)
\end{gathered}
$$

Where " $\mathrm{T}$ " is the reaction temperature in Celsius degrees; " $\mathrm{T}$ ref" is $100^{\circ} \mathrm{C}$; "t" is the reaction time in minutes; and 14.75 is the fitted value of an arbitrary constant. $\mathrm{pH}$ is measured before the reaction, at room temperature, having values of 4.92, 2.76, 2.03 and 1.76 for reaction using $0,50,200$ and $400 \mathrm{ppm}$ of $\mathrm{HCl}$, respectively. The most relevant results are summarized in Figure 7. As it can be observed, the maximum HMF productivity is obtained when CSF factor has a value close to 1.6-1.7. In order to obtain the maximum HMF concentration, this CSF factor

Table 4. Apparent and real kinetic constants obtained after fitting the experimental results to the model proposed. Results corresponding to reactions at $413 \mathrm{~K}$

\begin{tabular}{|c|c|c|c|c|c|c|c|c|c|c|}
\hline$k_{i}=k_{i}^{*} \cdot[\mathrm{HCl}]_{i}^{m_{i}}$ & $\begin{array}{c}\mathrm{HCl} \\
\text { (ppm) }\end{array}$ & $\mathrm{k}_{1}$ & $\mathrm{k}_{-1}$ & $\mathrm{k}_{2}$ & $\mathrm{k}_{3}$ & $\mathrm{k}_{4}$ & $k_{5}$ & $\mathrm{k}_{-5}$ & $\mathrm{k}_{7}$ & $r^{2}$ \\
\hline \multirow{4}{*}{$\begin{array}{l}\text { Apparent kinetic } \\
\text { constants* }^{*}\end{array}$} & 0 & $0.01 \pm 0.01$ & 0 & $0.42 \pm 0.13$ & $0.001 \pm 0.001$ & $0.53 \pm 0.11$ & $0.006 \pm 0.002$ & $0.07 \pm 0.01$ & & 0.993 \\
\hline & 50 & $0.12 \pm 0.04$ & $0.02 \pm 0.05$ & $0.17 \pm 0.03$ & $0.001 \pm 0.01$ & $0.49 \pm 0.04$ & $0.004 \pm 0.003$ & $0.04 \pm 0.02$ & $1.42 \pm 0.50$ & 0.92 \\
\hline & 200 & $0.43 \pm 0.01$ & $0.14 \pm 0.10$ & $0.69 \pm 0.10$ & $0.08 \pm 0.02$ & $1.13 \pm 0.08$ & $0.01 \pm 0.01$ & $0.19 \pm 0.14$ & $10.27 \pm 0.41$ & 0.97 \\
\hline & 400 & $0.53 \pm 0.03$ & $0.02 \pm 0.01$ & $1.15 \pm 0.17$ & $0.23 \pm 0.05$ & $1.33 \pm 0.09$ & $0.02 \pm 0.02$ & $0.22 \pm 0.04$ & $11.24 \pm 1.80$ & 0.92 \\
\hline \multicolumn{2}{|c|}{ Real kinetic constants ${ }^{* *}$} & 0.007 & 2.253 & 83.797 & $1 \cdot 10^{5}$ & 13.162 & 0.435 & 11.288 & $1.7 \cdot 10^{3}$ & - \\
\hline \multicolumn{2}{|c|}{ "m" exponential } & 0.7 & 1.1 & 0.9 & 2.8 & 0.5 & 0.7 & 0.8 & 1.1 & - \\
\hline \multicolumn{2}{|l|}{$r^{2}$} & 0.96 & 0.94 & 0.994 & 0.98 & 0.96 & 0.94 & 0.93 & 0.91 & - \\
\hline
\end{tabular}
with different mineral acid concentrations and using $\beta$-zeolite as heterogeneous catalyst.

* (units in $\mathrm{h}^{-1}$ except $\mathrm{k}_{4}$ and $\mathrm{k}_{7}$ that are in $\mathrm{L} \cdot \mathrm{mol}^{-1} \cdot \mathrm{h}^{-1}$ )

${ }^{* \star}\left(\right.$ units in $\mathrm{h}^{-1}\left(\mathrm{~L} \cdot \mathrm{mol}^{-1}\right)^{\mathrm{m}}$ except $\mathrm{k}_{4}$ and $\mathrm{k}_{7}$ that are in $\left.\mathrm{L} \cdot \mathrm{mol}^{-1} \cdot \mathrm{h}^{-1} \cdot\left(\mathrm{L} \cdot \mathrm{mol}^{-1}\right)^{\mathrm{m}}\right)$

the decomposition of HMF into acids. ${ }^{[37,38]}$

must be reached at the maximum temperature studied $\left(140^{\circ} \mathrm{C}\right)$. In fact, results obtained at lowest temperatures are, in all the cases, lower than those values obtained when using only homogenous catalysis (absence of zeolite, black asterisks in the figure). At $140^{\circ} \mathrm{C}$, the optimum situation can be obtained at large times (around $24 \mathrm{~h}$ ) if reaction is carried out with only $50 \mathrm{ppm}$ of $\mathrm{HCl}$, whereas this times strongly decreases if the mineral acid concentration increases to $200 \mathrm{ppm}$. In fact, the maximum HMF concentration is obtained at this conditions, after $5 \mathrm{~h}(0.06 \mathrm{~mol} \cdot \mathrm{L}$ $\left.{ }^{1}\right)$. Similar maximum concentration is obtained working with $400 \mathrm{ppm}$ of $\mathrm{HCl}$, but after only $3 \mathrm{~h}\left(0.063 \mathrm{~mol} \cdot \mathrm{L}^{-1}\right)$. Considering that, in both cases, these values correspond to $41 \%$ of selectivity, $200 \mathrm{ppm}$ of $\mathrm{HCl}$ and $5 \mathrm{~h}$ of reaction are chosen as the best reaction conditions in order to enhance the HMF production (differences are too low to justify the increase to $400 \mathrm{ppm}$ of $\mathrm{HCl}$ ). Concerning the LA, CSF higher than 2 are required to obtain a significant amount of this compound. In this case, the relevance of $\mathrm{HCl}$ concentration is more marked than for the $\mathrm{HMF}$, obtaining double amount of LA if results at 200 and $400 \mathrm{ppm}$ of $\mathrm{HCl}$ are compared. In summary, more than $0.11 \mathrm{~mol} \cdot \mathrm{L}^{-1}$ of $\mathrm{LA}$ are obtained after $24 \mathrm{~h}$ of reaction, with a global selectivity of $34 \%$, These conditions are chosen as the optimum ones to maximize the productivity of this platform molecule. In both
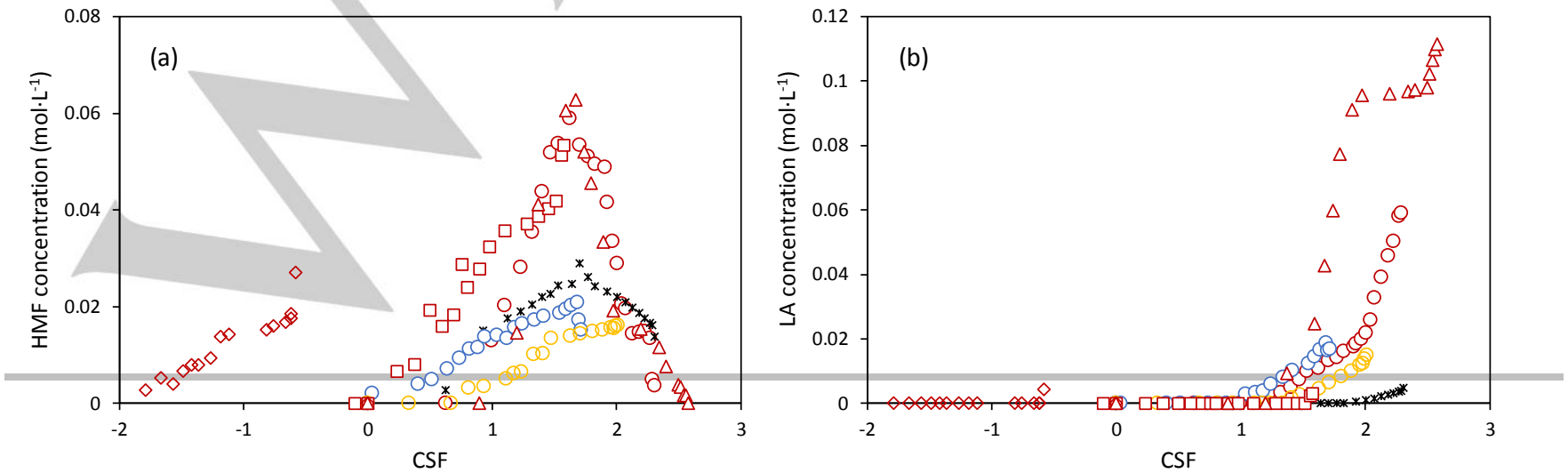

Figure 7: Analysis of (a) HMF and (b) LA productivity as function of the CSF factor. Data correspond to different concentration of $\mathrm{HCl}$ : 0 ppm (diamonds); 50 ppm (squares); 200 ppm (circles); 400 ppm (triangles). Results as function of the temperature: $393 \mathrm{~K}$ (blue); $403 \mathrm{~K}$ (yellow); $413 \mathrm{~K}$ (red). In order to make easier the comparison, results using only homogeneous catalysis $(200 \mathrm{ppm}$ of $\mathrm{HCl})$ at $140^{\circ} \mathrm{C}$ are also included $(*)$. 
cases, these results are significantly higher than those previously reported at similar severity factors when using only homogenous or heterogeneous catalysts. ${ }^{[7-10]}$

\section{Conclusions}

A clear improvement in the glucose valorisation to HMF and levulinic acid is obtained when heterogeneous and homogeneous catalysis are combined, obtaining promising results in aqueous medium. Beta zeolite is identified as the best material tested, with main effect in the improvement of glucose to fructose isomerization. A minimum temperature of $140^{\circ} \mathrm{C}$ is required to obtain significant amount of both platform molecules. After $24 \mathrm{~h}$ almost complete conversion (94\%) is obtained, with a significant decrease in the humins and $A H G$ production (main undesired reactions). Reaction time and $\mathrm{HCl}$ concentration can be modified to maximize HMF or LA, as required. Thus, maximum amount of HMF is obtained after $5 \mathrm{~h}$ with $200 \mathrm{ppm}$ of $\mathrm{HCl}$, whereas more severe conditions are required to enhance the LA $(24 \mathrm{~h}, 400 \mathrm{ppm}$ of $\mathrm{HCl})$. Under these conditions, both platform molecules are the main product obtained. A complete kinetic model is proposed, combining the effect of both catalysis. A clear decrease in the activation energy of main steps is obtained, with values $30-40 \%$ lower than those previously proposed when using only mineral acid.

\section{Experimental Section}

\section{Materials}

D-(+)-glucose ( $\geq 99.5 \%)$ and sodium hydrogen carbonate $(\geq 99.7 \%)$ were purchased from Panreac Applichem. 5-(Hydroxymethyl)furfural $(\geq 99 \%)$, formic acid $(98 \%)$ and levulinic acid $(98 \%)$ for HPLC calibration were acquired from Sigma-Aldrich, and hydrochloric acid (37\%) was purchased from Fisher Chemical. $\beta$-zeolite $\mathrm{CP} 814 \mathrm{E}\left(\mathrm{SiO}_{2} / \mathrm{Al}_{2} \mathrm{O}_{3}=25\right)$, ZSM-5 CBV2314 $\left(\mathrm{SiO}_{2} / \mathrm{Al}_{2} \mathrm{O}_{3}=23\right)$ and Y-zeolite CBV712 $\left(\mathrm{SiO}_{2} / \mathrm{Al}_{2} \mathrm{O}_{3}=12\right)$ were purchased from Zeolyst International. Zeolites were activated by a heat treatment at $823 \mathrm{~K}$ for removing the $\mathrm{NH}_{4}{ }^{+}$functional groups of the commercial zeolites, obtaining the proton forms that are active for this reaction. This treatment has been previously reported in the literature. ${ }^{[44]}$

\section{Reaction system}

Reactions were carried out in a $0.5 \mathrm{~L}$ stirred batch autoclave reacto (Autoclave Engineers EZE seal) with a backpressure regulator and a PID temperature controller. In a typical experiment, $0.175 \mathrm{~L}$ of an aqueous solution of $5.83 \mathrm{~g}$ of $\mathrm{D}$-(-)-glucose and $0.466 \mathrm{~g}$ of $\beta$-zeolite were used. Four different $\mathrm{HCl}$ concentrations were studied $(0-400 \mathrm{ppm}$, corresponding to feed $\mathrm{pH}$ between 4.92 and 1.76) and the influence of reaction temperature was also analysed (from 393 to $413 \mathrm{~K}$ ). Once reached the desired temperature, $25 \mathrm{~mL}$ of a solution of hydrochloric acid was added resulting the acid concentration corresponding to each experiment. Air was purged with $\mathrm{N}_{2}$ and dehydration was carried out with 10 bar of $\mathrm{N}_{2}$ with a stirring of 600 rpm for 24 hours.

\section{Analysis}

Samples were taken from the sampling port, filtered using $0.45 \mu \mathrm{m}$ Nylon syringe filters and diluted in a 1:20 ratio. The reactants were quantified by HPLC (1200 Series, Agilent) using a refraction index detector (G1362A $\mathrm{Rl}$ ). A Hi-Plex $\mathrm{H}$ Column (Agilent) was used as stationary phase, whereas $0.6 \mathrm{~mL} / \mathrm{min}$ of a $5 \mathrm{mM} \mathrm{H}_{2} \mathrm{SO}_{4}$ solution was chosen as the mobile phase. With these data, selectivities are calculated according to the following equation:

$$
\mathrm{S}_{\mathrm{i}}=\mathrm{n}_{\mathrm{i}} \cdot \mathrm{mol}_{\mathrm{i}} / \sum\left(\mathrm{n}_{\mathrm{i}} \cdot \mathrm{mol}_{\mathrm{i}}\right)
$$

where " $n_{i}$ " correspond to the number of carbon of the component "i"; and the denominator.

\section{Kinetic modelling}

The kinetic model is based on the assumption that the system can be described as an ideal stirred batch reactor (BR). Mass balance for a given compound follows a differential equation considering both homogeneous and heterogeneous kinetic contributions. By combining the reactor model and the power-law rate expressions, the complete model is obtained. Equations obtained were implemented and solved in Scientist ${ }^{\circledR}$ 3.0 Software (MicroMath) and experimental results were fitted to model proposed considering the least squares methodology.

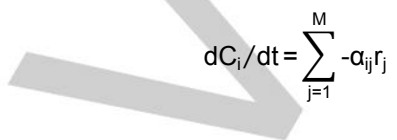

being " $\mathrm{C}_{\mathrm{i}}$ ", concentration of "i" component $\left(\mathrm{mol} \mathrm{L}^{-1}\right)$; $\alpha_{i j}$, stoichiometric coefficient of component "i" on the reaction " $\mathrm{j}$ "; " $\mathrm{r}_{\mathrm{j}}$ ", reaction rate for the reaction "j" ( $\left.\mathrm{mol} \mathrm{L}^{-1} \mathrm{~s}^{-1}\right)$.

$$
\mathrm{r}_{\mathrm{j}}=\mathrm{k}_{\mathrm{jHo}} \prod_{\mathrm{k}=1}^{\mathrm{N}} \mathrm{C}_{\mathrm{k}}^{\mathrm{n}_{\mathrm{kHo}}}+\mathrm{k}_{j H e} \prod_{\mathrm{k}=1}^{\mathrm{N}} \mathrm{C}_{\mathrm{k}}^{\mathrm{n}_{\mathrm{kHe}}}
$$

being " $\mathrm{k}_{\mathrm{jHo}}$ ", rate constant of "j" reaction corresponding to homogeneous reaction; " $\mathrm{C}_{k}$," concentration of " $\mathrm{k}$ " component $\left(\mathrm{mol} \mathrm{L}^{-1}\right)$; " $\mathrm{n}_{\mathrm{kH}}$ ", reaction order of " $\mathrm{k}$ " component corresponding to homogeneous reaction; " $\mathrm{k}_{\mathrm{jHe}}$ ", rate constant of "j" reaction corresponding to heterogeneous reaction; " $\mathrm{n}_{\mathrm{kHe}}$ ", reaction order of " $\mathrm{k}$ " component corresponding to heterogeneous reaction.

\section{Acknowledgements}

This work has been financed by Research Projects of the Regional Government of Asturias (project reference GRUPIN14078) and Spanish Ministry of Economy and Competitiveness (CTQ2014-52956-C3-1-R and CTQ2017-89443-C3-2-R). Diego Garcés thanks the Government of the Principality of Asturias for a Ph.D. fellowship (Severo Ochoa Program).

Keywords: kinetics $\bullet$ biomass $\bullet$ zeolites $\bullet$ bio-platform molecules - biorefinery

[1] a) J. Cueto, L. Faba, E. Díaz, S. Ordóñez, Appl. Catal. B 2017, 201 221-231; b) D.M. Alonso, S.G. Wettstein, M.A. Mellmer, E.I. Gurbuz, J.A. Dumesic, Energy Environ. Sci. 2013, 6, 76-80; c) F.S. Asghari, H. Yoshida, Ind. Eng. Chem. Res. 2007, 46, 7703-7710. d) L. Faba, E. Díaz, S. Ordóñez, Renew. \& Sust. Energy Rev. 2015, 51, 273-287

[2] S.S. Chen, T. Maneerung, D.C.W. Tsang, Y.S. Ok, C-H. Wang, Chem Eng. J. 2017, 328, 246-273.

[3] J.J. Bozell, G.R. Petersen, Green Chem. 2010, 12, 539-554.

[4] R. Weingarten, J. Cho, R. Xing, W.C. Conner, G.W. Huber ChemSusChem 2012, 5, 1280-1290.

[5] R. Rinaldi, F. Schüth, ChemSusChem 2009, 2, 1096-1107.

[6] B.F.M. Kuster, Starch 1990, 42, 314-321. 
[7] L. Yang, G. Tsilomelekis, S. Caratzoulas, D.G. Vlachos, ChemSusChem 2015, 8, 1334-1341.

[8] B. Girisuta, K. Dussan, D. Haverty, J.J. Leahy, M.H.B. Hayes, Chem. Eng. J. 2013, 217, 61-70.

[9] a) J.N. Chheda, Y. Román-Leshkov, J.A. Dumesic J.A. Green Chem 2007, 9, 342-350; b) V. Choudhary, R.I. Burnett, D.G. Vlachos, S.I. Sandler, J. Phys.Chem. C 2012, 116, 5116-5120.

[10] A.T. Pedersen, R. Ringborg, T. Grotkjaer, S. Pedersen, J.M. Woodley, Chem. Eng. J. 2015, 273, 455-464.

[11] Y. Román-Leshkov, J.N. Chheda, J.A. Dumesic, Science 2006, 312 1933-1937.

[12] a) J. Fu, X. Xu, x. Lu, X. Lu, Ind. Eng. Chem. Res. 2016, 55, 1104411051 ; b) S. Hu, Z. Zhang, J. Song, Y. Zhou, B. Hand, Green Chem 2009, 11, 1746-1749; c) C. Li, Z.K. Zhao, Adv. Synth. Catal. 2007, 349, 1847-1850.

[13] a) R.M. Musau, R.M. Munavu, Biomass 1987, 13, 67-74; b) H.B. Zhao, J.E. Holladay, J.H. Kwak, Z.C.J. Zhang, Science 2007, 316,1597-1600 c) L. Qi, Y.W. Mui, S.W. Lo, M.Y. Lui, g.R. Akien, I.T. Horváth, ACS Catal. 2014, 4, 1470-1477.

[14] Y. Román-Leshkov, J.A. Dumesic, Top. Catal. 2009, 525, 297-303.

[15] R. Dornath, W. Fan, Microp. Mesop. Mater. 2014, 191, 10-17.

[16] J.C. Serrano-Ruiz, J.A. Dumesic, Energy Environ. Sci. 2011, 4, 83-99.

[17] M. Moreno-Recio, J. Santamaría-González, P. Marieles-Torres, Chem. Eng. J. 2016, 303, 22-30.

[18] a) T. Dallas Swift, C. Bagia, V. Choudhary, G. Peklaris, V. Nikolakis, D.G. Vlachos, ACS Catal. 2013, 4, 259-267; b) M. Moliner, Y. RománLeshkov, M.E. Davis, Proc. Natl. Acad. Sci. USA 2010, 107, 61664 6168; c) A. Osatiashtiani, A.F. Lee, M. granollers, D.R. brown, L. Olivi, G. Morales, J.A. Melero, K. Wilson, ACS Catal. 2015, 5, 4345-4352.

[19] I. Jiménez Morales, M. Moreno Recio, J. Santamaría González, P. Maireles Torres, A. Jiménez López, Appl. Catal. B 2015, 164, 70-76.

[20] a) E. Nikolla, Y. Román-Leshkov, M. Moliner, M.E. Davis, ACS Catal. 2011, 1, 408-410; b) Q. Hou, M. Zhen, L. Liu, Y. Chen, F. Huang, S. Zhang, W. Li, M. Ju, Appl. Catal. B 2018, 224, 183-193.

[21] D. Garcés, E. Díaz, S. Ordóñez, Ind. Eng. Res. 2017, 56, 5221-5230.

[22] S.J. Dee, A.T. Bell, ChemSusChem, 2011, 4, 1166-1173.

[23] A. Aho, N. Kumar, K. Eränen, T. Salmi, M. Hupa, D.Yu Murzin, Fuel, 2008, 87, 2493-2501

[24] R. Xu, W. Pang, J. Yu, Q. Huo, J. Chen in Chemistry of zeolites and related propous materials: synthesis and structure, Wiley, United States, 2007, pp.44-56.

[25] M.J. Taylor, L.J. Durndell, M.A. Isaacs, C.M.A. Parlett, K. Wilson, A.F Lee, G. Kyriakou, Appl. Catal. B 2016, 180, 580-585.

[26] H. Kimura, M. Nakahara, N. Matubayasi, J. Phys. Chem. A 2011, 115, 14013-14021.

[27] C.P. Locas, V. Yaylayan, J. Agric. Food Chem. 2008, 56, 6717-6723.

[28] Y.J. Pagán-Torres, T. Wang, J.M.R. Gallo, B.H. Shanks, J.A. Dumesic, ACS Catal. 2012, 2, 1925-1930.

[29] W. Weiqi, W. Shubin, Fuel 2018, 225, 311-321.

[30] H.M. Pilath, M.R. Nimlos, A. Mittal, M.E. Himmel, D.K. Johnson, J. Agric. Foo Chem. 2010, 58, 6131-6140.

[31] R.J. van Putten, J.C. van Der Waal, E. Jong, C.B. Rasrendra, H.J. Heeres, J.G. de Vries, Chem. Rev. 2013, 113, 1499-1597.

[32] S. Patil, C. Lund, Energy Fuels 2011, 25, 4745-4755.

[33] P. Dornath, W. Fan, Micropour. Mesopour. Mat. 2014, 191, 10-17

[34] T. Swift, C. Bagia, V. Choudhary, G. Peklaris, V. Nikolakis, D.G. Vlachos, ACS Catal. 2013, 4, 259-267.

[35] R.J. van Putten, J.N. Soetedjo, E.A. Pidko, J.C. van der Waal, E.J. Hensen, E. de Jong, H.J. Heeres, ChemSusChem 2013, 6,1681-1687.

[36] M. Bicker, J. Hirth, H. Vogel, Green Chem. 2003, 5, 280-284.

[37] B. Girisuta, L.P.B.M. Janssen, H.J. Heeres, Ind. Eng. Chem. Res. 2007 46, 1696-1708.

[38] B. Girisuta, L.P.B.M. Janssen, H.J. Heeres, Green Chem. 2006, 8, 701 709

[39] R.P. Overend, E. Chornet, Philos. Trans. R. Soc. Lond. 1987, 321, 523536 .
[40] P.K. Keshov, C. Bonoth, A. Anthappayudem, V.R. Linga, B. Bhukya Ind. Crop. Prod. 2015, 125, 462-467.

[41] R.Y. Spitzer, V. Mau, A. Gross, J. Cleaner Prod. 2018, 205, 955-963.

[42] J.K. Ko, Y. Kim, E. Ximenes, M.R. Ladish, Biotechnol. Bioeng. 2015 $112,252-262$

[43] H.S. Kim, S.K. Kim, G.T. Jeong, J. Ind. Eng. Chem. 2018, 63, 48-56.

[44] R.E. Patet, M. Kohle, R.F. Lobo, S. Caratzoulas, D.G. Vlachos, J. Phys. Chem. C 2017, 121, 13666-13679. 
Entry for the Table of Contents (Please choose one layout)

Layout 1:

\section{FULL PAPER}

Cooperation in catalysis: The presence of a heterogeneous acid catalyst, as zeolite beta, promotes the transformation of glucose into hydroximethylfurfural or levulinic acid (depending on reaction conditions). Specifically, the presence of Lewis acid sites increases the reaction rate of the glucose isomerization into fructose, a more reactive hexose.

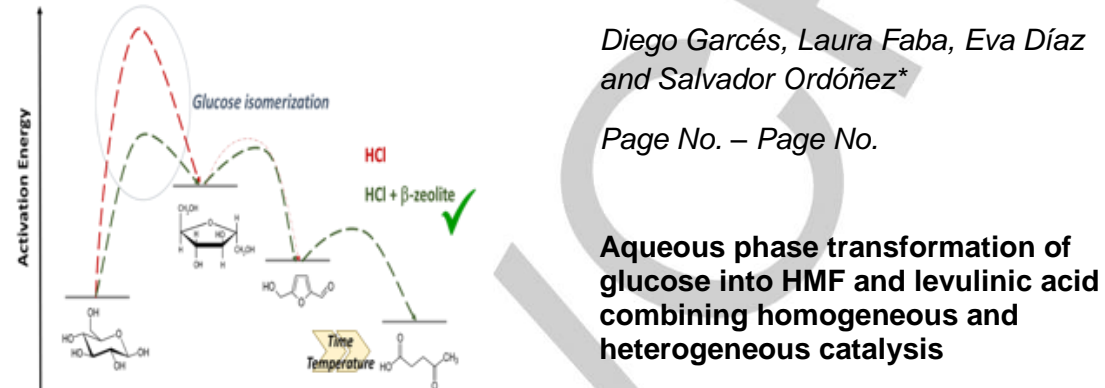

Diego Garcés, Laura Faba, Eva Díaz

heterogeneous catalysis 